\title{
The Effect of Using Sinusoidal Profile in Fins on Thermal Performance
}

\author{
Hakan Sertel $^{1 *}$, Kemal Bilen ${ }^{2}$ \\ ${ }^{1}$ Eskişehir Osmangazi University, Faculty of Engineering and Architecture, Department of Mechanical Engineering, Eskişehir \\ 26480, Turkey \\ ${ }^{2}$ Department of Mechanical Engineering, Faculty of Engineering and Natural Sciences, Ankara Y1ldırım Beyazıt University, \\ Ankara 06010, Turkey
}

Corresponding Author Email: hakansertel@ windowslive.com

https://doi.org/10.18280/ijht.370310

Received: 21 May 2019

Accepted: 12 September 2019

\section{Keywords:}

fins, sinusoidal profile, shooting method, Runge Kutta, CFD

\begin{abstract}
The aim of the present study is to investigate the effect of using sinusoidal profile in fins on temperature distribution and heat transfer enhancement. The longitudinal fins of rectangular, triangular, wavy rectangular and wavy triangular profiles are studied using analytical and numerical solution techniques. In addition to known profiles, wavy triangular longitudinal fins are proposed as a new fin configuration. The temperature distribution of wavy triangular fins is obtained using Shooting Method along with Cash-Karp Runge Kutta Method. Besides, CFD analyses in which laminar flow regime is concerned at different air velocities is utilized for the comparison of heat transfer rates and convective heat transfer coefficients. CFD results show that heat transfer convection coefficient values for wavy rectangular fins are the lowest ones among the investigated fins. CFD and analytical results indicate that for lower convective coefficients, the dominancy of triangular fins having wave form on plate type triangular fins is more noticeable than the dominancy of wavy rectangular fins on plate type rectangular fins. Also, further heat transfer augmentation can be provided for wavy triangular fins at higher amplitude lengths. For instance, heat transfer enhancement by $15.3 \%$ can be achieved using wavy triangular fins with thickness of $2 \mathrm{~cm}$, amplitude length of $0.21 \mathrm{~cm}$ and three-wave profile in comparison with triangular fins having same dimensions.
\end{abstract}

\section{INTRODUCTION}

In different engineering fields in which high amount of heat removal is concerned, extended surfaces called fins can be implemented to related devices in order to provide heat transfer augmentation. Particularly, heat exchangers, gas turbines, diverse electronic components and other many thermal systems are the areas of applications where various type of fins are used [1,2].

Although fins are one of the passive techniques used on the purpose of heat transfer enhancement, many researchers have studied on improving heat dissipation from fins using different profiles. For instance, in Ref. [3], the writers analytically investigated a triangular fin by embedding its partial part to a wall. In this study, they obtained significant heat transfer enhancement at wall Biot number of 1.54 in comparison to both plain wall without fins and plain wall with ordinary triangular fins. Furthermore, exponential fins were studied in Ref. [4] and Ref. [5]. In the study of Ref. [4], the writer stated that thermal efficiency of exponential straight fins is greater than that of rectangular, triangular and parabolic straight fins whereas triangular and parabolic pin fins are thermally more efficient than exponential pin fins. As another geometrical shape, fins having sinusoidal wave form have been studied. For instance, in Ref. [6], various fins having different form of sinusoidal profiles studied. The writer compared the wavy fins in terms of fin efficiency and two different performance indicators. As another study concerning wavy fins, a numerical study in which fins having sinusoidal profile were analyzed at different wave numbers in Ref. [7]. Moreover, wavy fins were experimentally investigated under the heat transfer mechanisms of both natural convection and forced convection. In Ref. [8], wavy fins mounted to horizontally oriented base plate were investigated under natural convection and compared with rectangular fins. The writers reveal that the highest Nusselt number value was attained for wavy fins having the lowest amplitude due to the blockage effect to fluid motion in higher amplitudes. In Ref. [9], wavy fins with and without crosscut were investigated experimentally at different phase shift angles. According to the results, the writers stated that the wavy fins with crosscut dominated on those without crosscut in terms of thermal performance while pressure drop performance was better for those without crosscut.

CFD (computational fluid dynamics) is a common numerical method that can be used in the studies where combined heat transfer and fluid flow are investigated such as fin analysis. In Ref. [10], the writer numerically examined straight rectangular fins for two different cases which are fins with constant thermal conductivity and temperature dependent conductivity using CFD software package. In addition to CFD investigation, researchers have experimentally studied thermal performance of extended surfaces. To illustrate, in Ref. [11], the writers determined convective heat transfer coefficient values for the flow over plate fins using numerical and experimental techniques. The writers attained smaller convective heat transfer coefficient values for smaller fin pitch lengths.

For the comparison of different fin configurations in terms 
of thermal performance, some studies have been conducted in various conditions. For instance, in Ref. [12], the writers studied different convective-radiative fins having internal heat generation in a case that thermal conductivity, convective heat transfer coefficient, surface emissivity and internal heat generation were all temperature dependent. They used differential transformation method (DTM) and integral approach to acquire temperature distribution and fin efficiency, respectively. Similarly, in Ref. [13], the writers investigated convective-radiative longitudinal fins having rectangular, trapezoidal, and concave parabolic profiles. They did not account for non-uniform internal heat generation, unlike the former study.

Shooting Method in conjunction with Runge Kutta Method is one of the numerical strategies used for the solution of boundary value problems. In Ref. [14], these methods were used to solve a non-linear problem related to a straight fin with uniform cross section in case of temperature dependent convective heat transfer coefficient. Also, the third order nonlinear ordinary differential equation which governs laminar boundary layer problem was solved in Ref. [15] with the help of Shooting and Runge Kutta Methods.

The present study involves analytical and numerical investigations of rectangular, triangular, wavy rectangular and wavy triangular longitudinal fins. The governing differential equation concerning wavy triangular fins is solved by means of Shooting Method together with Runge Kutta Method to obtain temperature distribution and heat transfer rate. For wavy longitudinal fins, results are obtained for different amplitude lengths and wave numbers. Furthermore, CFD analyses are performed through the software, ANSYS Fluent. In the analyses, laminar flow regime is utilized. Overall results for the investigated fins are compared and the superiorities and weaknesses of the fins in terms of different parameters are presented.

\section{SOLUTION METHOD}

\subsection{Analytical analysis of the problem}

Before the analytical investigation of the problem, some necessary assumptions are put forward. The equations concerning the fin geometries are constructed based on the following assumptions;

- $\quad$ Steady-state heat transfer, $\frac{\partial()}{\partial \tau}=0$.

- One-dimensional heat transfer (in $x$-direction).

- Thermal conductivity of the fin material is constant.

- Convective heat transfer coefficient is constant.

- The thickness of the fins is so small in comparison to their width that heat transfer from lateral surfaces might be neglected.

- The ambient temperature is constant.

- The base temperature of the fin is uniform.

- There is no contact resistance between the fin base and primary surface.

- There is no heat generation inside the fins.

General differential equation for fins that is valid for steadystate and one-dimensional heat transfer is represented by;

$$
\frac{d^{2} T}{d x^{2}}+\left(\frac{1}{A_{c}} \frac{d A_{c}}{d x}\right) \frac{d T}{d x}-\left(\frac{1}{A_{c}} \frac{h}{k} \frac{d A_{s}}{d x}\right)\left(T-T_{a}\right)=0
$$

$T$ and $T_{a}$ are defined as temperature within the fin and ambient temperature, respectively. $A_{\mathrm{c}}$ and $A_{s}$ are depicted as cross-sectional area and surface area, respectively. Thermal conductivity of the fin material and convective heat transfer coefficient are represented by $k$ and $h$, respectively. Also, the thickness, length and width of the fins are denoted by $t, L$ and $W$, respectively as shown in Figure 1. For the wavy rectangular fins, the coordinate following the sinusoidal profile, scoordinate is used to form the governing equation.

Non-dimensional expressions are given by;

$$
\xi=\frac{x}{L}, \quad \delta=\frac{s}{S_{r}}, \quad \Theta(\xi)=\frac{T(x)-T_{a}}{T_{b}-T_{a}}
$$

where, $\xi$ and $\delta$ are the non-dimensional coordinates and $\Theta$ is the dimensionless temperature distribution.

a)

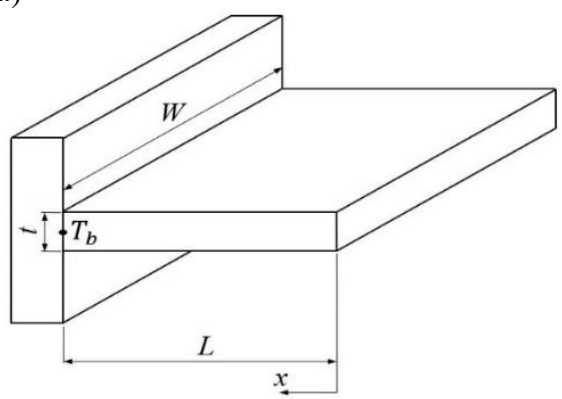

b)

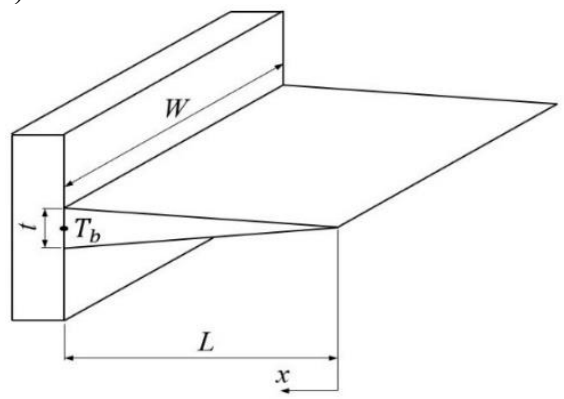

c)

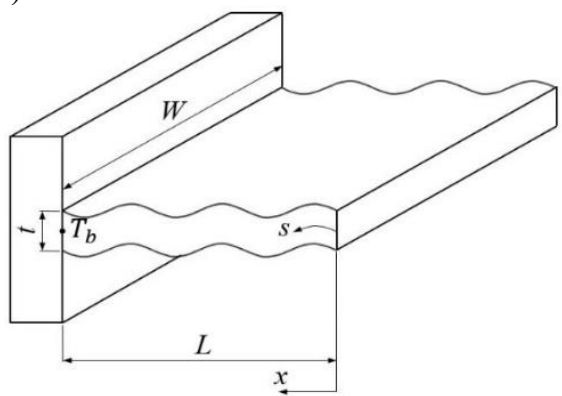

d)

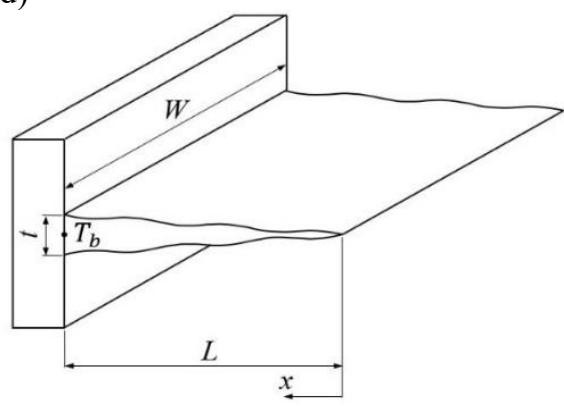

Figure 1. Schematic views of a) rectangular, b) triangular, c) wavy rectangular, d) wavy triangular longitudinal fins 
As boundary conditions, constant base temperature and heat convection at fin tip are determined and they are depicted in terms of dimensionless parameters in Eq. (3);

$$
\left.\frac{k}{L} \frac{d \Theta}{d \xi}\right|_{\xi=0}=h \Theta(0), \quad \Theta(1)=1
$$

Another parameter, fin performance factor is defined as;

$$
m=\sqrt{\frac{2 h}{k t}}
$$

Using the boundary conditions and general differential equation that governs temperature distribution within a fin, four type of fin geometry are analytically analyzed. Wavy profiled fins are investigated in terms of heat transfer enhancement and their superiority to plate type fins having rectangular and triangular geometries is put forward. The advantage of using sinusoidal profile is that there is no need for additional material for increasing the surface area to contribute to heat transfer augmentation because sinusoidal wave makes peak up and down.

\subsubsection{Rectangular longitudinal fins}

The governing equation for rectangular fins, temperature distribution equation and heat transfer rate are formulated as follows [16].

$$
\begin{gathered}
\frac{d^{2} \Theta}{d \xi^{2}}-m^{2} L^{2} \Theta=0 \\
\Theta(\xi)=\frac{\cosh (m L \xi)+\frac{h}{m k} \sinh (m L \xi)}{\cosh (m L)+\frac{h}{m k} \sinh (m L)} \\
q_{f}=k W t\left(T_{b}-T_{a}\right) m \frac{\sinh (m L)+\frac{h}{m k} \cosh (m L)}{\cosh (m L)+\frac{h}{m k} \sinh (m L)}
\end{gathered}
$$

\subsubsection{Triangular longitudinal fins}

The solution of the differential equation for triangular longitudinal fins yields Bessel equations through which temperature distribution is obtained. The related equations are represented by the following expressions [2].

$$
\begin{aligned}
& \xi \frac{d^{2} \Theta}{d \xi^{2}}+\frac{d \Theta}{d \xi}-m^{2} L^{2} \Theta=0 \\
& \Theta(\xi)=\frac{I_{0}(2 m L \sqrt{\xi})}{I_{0}(2 m L)} \\
& q_{f}=\frac{2 h W\left(T_{b}-T_{a}\right) I_{1}(2 m L)}{m I_{0}(2 m L)}
\end{aligned}
$$

\subsubsection{Wavy rectangular longitudinal fins}

The profile function for wavy rectangular fins which are also studied in Ref. [6] is given as;

$$
F_{r}(\xi)=\beta \sin (2 \pi n \xi)
$$

where, $\beta$ is the length of amplitude and $n$ is the number of waves. The arc length of the sinusoidal profile, $S_{r}$ can be defined by the formula;

$$
S_{r}=L \int_{0}^{1} \sqrt{1+\left[\frac{2 \pi n \beta}{L} \cos (2 \pi n \xi)\right]^{2}} d \xi
$$

If non-dimensional form of Eq. (1) is adapted to wavy rectangular longitudinal fins, the resulting differential equation becomes;

$$
\frac{d^{2} \Theta}{d \delta^{2}}-m^{2} S_{r}^{2} \Theta=0
$$

Other corresponding equations can be stated as;

$$
\begin{gathered}
\Theta(\delta)=\frac{\cosh \left(m S_{r} \delta\right)+\frac{h}{m k} \sinh \left(m S_{r} \delta\right)}{\cosh \left(m S_{r}\right)+\frac{h}{m k} \sinh \left(m S_{r}\right)} \\
q_{f}=k W t\left(T_{b}-T_{a}\right) m \frac{\sinh \left(m S_{r}\right)+\frac{h}{m k} \cosh \left(m S_{r}\right)}{\cosh \left(m S_{r}\right)+\frac{h}{m k} \sinh \left(m S_{r}\right)}
\end{gathered}
$$

Another parameter that gives the ratio of heat dissipation of the wavy rectangular fins to that of the rectangular fins is the performance ratio, $\phi_{\mathrm{r}}$ and it is formulated by;

$$
\phi_{r}=\frac{q_{f, \text { wavy-rec }}}{q_{f, r e c}}
$$

\subsubsection{Wavy triangular longitudinal fins}

For the heat transfer augmentation, wavy triangular fins are designed as a new fin configuration. The parameters to help constructing the governing eqution are given in this section. The profile function for wavy triangular longitudinal fins is given as;

$$
F_{r}(\xi)=\frac{t}{2} \xi+\beta \sin (2 \pi n \xi)
$$

The profile function given in Eq. (17) indicates actually an inclined sine curve. The arc length of the sinusoidal profile is formulated by;

$$
S_{t}=L \int_{0}^{1} \sqrt{1+\left[\frac{t}{2 L}+\frac{2 \pi n \beta}{L} \cos (2 \pi n \xi)\right]^{2}} d \xi
$$

The cross-sectional area and the surface area of the wavy triangular fins are given as follows;

$$
A_{s}(\xi)=2 W L \int_{0}^{\xi} \sqrt{1+\left[\frac{t}{2 L}+\frac{2 \pi n \beta}{L} \cos (2 \pi n \omega)\right]^{2}} d \omega
$$




$$
A_{c}(\xi)=W[t \xi+2 \beta \sin (2 \pi n \xi)]
$$

Using the equations given above, the differential equation can be established as the following expression;

$$
\begin{aligned}
& \frac{d^{2} \Theta}{d \xi^{2}}+\left[\frac{t+4 \pi n \beta \cos (2 \pi n \xi)}{t \xi+2 \beta \sin (2 \pi n \xi)}\right] \frac{d \Theta}{d \xi} \\
& -\frac{m^{2} L^{2}}{\xi+\frac{2 \beta}{t} \sin (2 \pi n \xi)} \sqrt{1+\left[\frac{t}{2 L}+\frac{2 \pi n \beta}{L} \cos (2 \pi n \xi)\right]^{2}} \Theta=0
\end{aligned}
$$

Since the analytical solution of Eq. (21) is not possible, it is solved numerically. Heat dissipation equation for wavy triangular longitudinal fins can be stated as follows;

$$
q_{f}=\left.k W t \frac{\left(T_{b}-T_{a}\right)}{L} \frac{d \Theta}{d \xi}\right|_{\xi=1}
$$

The performance ratio, $\phi_{t}$ which gives the ratio of heat transfer rate of wavy triangular fins to that of triangular fins is represented by;

$$
\phi_{t}=\frac{q_{f, w a v y-t r i .}}{q_{f, t r i .}}
$$

Since triangular geometry has decreasing cross-sectional area from base to tip, to determine amplitude length and number of waves might be a limitation particularly for thin fins. However, for relatively thick fins, the surface area might be increased since amplitude length and the number of waves can be increased more easily.

\subsection{Numerical analysis of the problem}

The numerical solution procedure of the study can be divided into two parts. The numerical technique to solve the differential equation concerning wavy triangular fins is constituted based on Shooting and Runge Kutta Methods. The second part comprises the CFD analysis of the longitudinal fins.

In order to solve the governing equation of wavy triangular fins, Shooting Method is used. Before applying the related numerical method, the general non-dimensional differential equation for fins is stated as;

$$
\frac{d^{2} \Theta}{d \xi^{2}}+B \frac{d \Theta}{d \xi}-C \Theta=0
$$

where, $B$ and $C$ are the coefficients which vary depending on the differential equation concerning the longitudinal fin to which the method is applied. The Shooting Method constructs a relationship between a boundary value problem and equivalent initial value problems. Implementing Shooting Method to Eq. (24), the second order linear differential equation is reduced to a couple of first order linear differential equations as seen in the following statement.

$$
\begin{aligned}
& \frac{d \Theta}{d \xi}=w \\
& \frac{d w}{d \xi}=-B w+C \Theta
\end{aligned}
$$

where, $w$ is the temperature gradient. The initial conditions for the system of first order differential equations are given as;

$$
\begin{aligned}
& \Theta(1)=1, \quad w(0)=\frac{h L}{k} \Theta(0) \\
& \text { continuity : } \quad \frac{\partial u}{\partial x}+\frac{\partial v}{\partial y}+\frac{\partial w}{\partial z}=0 \\
& x \text {-momentum }: \quad \rho\left(u \frac{\partial u}{\partial x}+v \frac{\partial u}{\partial y}+w \frac{\partial u}{\partial z}\right)=-\frac{\partial P}{\partial x} \\
& +\mu\left(\frac{\partial^{2} u}{\partial x^{2}}+\frac{\partial^{2} u}{\partial y^{2}}+\frac{\partial^{2} u}{\partial z^{2}}\right) \\
& y \text {-momentum: } \quad \rho\left(u \frac{\partial v}{\partial x}+v \frac{\partial v}{\partial y}+w \frac{\partial v}{\partial z}\right)=-\frac{\partial P}{\partial y} \\
& +\mu\left(\frac{\partial^{2} v}{\partial x^{2}}+\frac{\partial^{2} v}{\partial y^{2}}+\frac{\partial^{2} v}{\partial z^{2}}\right) \\
& z \text {-momentum }: \quad \rho\left(u \frac{\partial w}{\partial x}+v \frac{\partial w}{\partial y}+w \frac{\partial w}{\partial z}\right)=-\frac{\partial P}{\partial z} \\
& +\mu\left(\frac{\partial^{2} w}{\partial x^{2}}+\frac{\partial^{2} w}{\partial y^{2}}+\frac{\partial^{2} w}{\partial z^{2}}\right) \\
& \text { energy: } \quad \rho c_{p}\left(u \frac{\partial T}{\partial x}+v \frac{\partial T}{\partial y}+w \frac{\partial T}{\partial z}\right)=-\frac{\partial P}{\partial x} \\
& +k_{\text {fluid }}\left(\frac{\partial^{2} T}{\partial x^{2}}+\frac{\partial^{2} T}{\partial y^{2}}+\frac{\partial^{2} T}{\partial z^{2}}\right)
\end{aligned}
$$

To solve the first order differential equations, Cash-Karp Runge Kutta Method [17] is used. The numerical method is firstly applied to the differential equation for triangular fin to observe whether the numerical method gives the desired accuracy.

For the CFD analysis of the longitudinal fins, a flow domain into which the fins are placed is determined along with its dimensions and demonstrated in Figure 2. As boundary conditions, the surfaces of $C, E$, and $F$ are determined as 'velocity-inlet'. To be more precise, velocity is defined in the tangential direction of these surfaces. "Adiabatic wall boundary condition' is applied to surface $\mathrm{D}$. Also, the surface $A$ is 'inlet' for the air flow which is in the $z$-direction whereas surface $B$ represents the 'outlet'. Besides, the boundary conditions for the fins are; $(i)$ constant temperature at the fin base and (ii) convective fin tip. The fins to be analyzed are mounted on surface $D$ of the flow domain and they are centered over $y$-coordinate. The length from inlet to the center of the fin in z-direction is $5 \mathrm{~W}$, while $20 \mathrm{~W}$ is the remaining length up to the outlet.

The continuity, momentum and energy equations for 3D, incompressible and steady flow are given in Eq. (27) [18]. Also, body forces are not included in the related equations.

'The SIMPLE algorithm' is chosen for the solution procedure of the problem and for the discretization of energy and momentum equations, 'second order upwind scheme' is 
determined through the CFD package. As mesh type, tetrahedral elements are used.

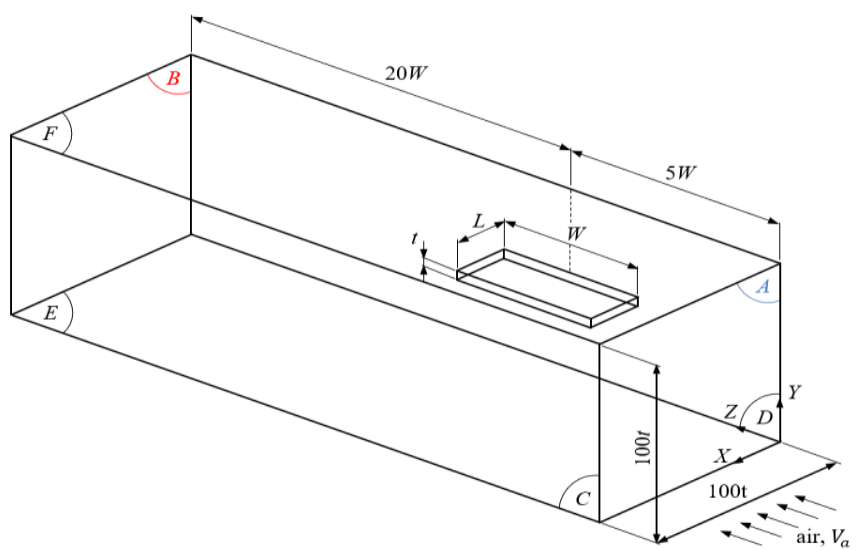

Figure 2. The flow domain used in CFD analyses

In the direction of the related parameters, the analyses are carried out at different air velocities in case that the temperatures for the fin base and air are $200{ }^{\circ} \mathrm{C}$ and $25^{\circ} \mathrm{C}$, respectively. The material of the fins is chosen as aluminum alloy with a thermal conductivity of $180 \mathrm{~W} /(\mathrm{m} \cdot \mathrm{K})$. Aluminum is one of the most preferable fin materials due to the properties of it that it is highly thermal conductive, which assists heat dissipation and it is also a lightweight material. Besides, aluminum can be easily manufactured [8], and this is good for particularly manufacturing of wavy fins. Also, the thermophysical properties of air are determined at the film temperature which is the half of the sum of fin base temperature and ambient temperature. It is represented by the following equation.

$$
T_{f}=\frac{T_{a}+T_{b}}{2}
$$

The properties calculated at the film temperature are given in Table 1 as density, $\rho$, specific heat, $c_{p}$, dynamic viscosity, $\mu$, thermal conductivity, $k$ and Prandtl number $P r$.

Table 1. The thermophysical properties of air at the film temperature

\begin{tabular}{lllll}
\hline $\mathbf{\rho}, \mathbf{~ k g} / \mathbf{m}^{3} \mathbf{c}_{\mathbf{p}}, \mathbf{J} /(\mathbf{k g} \cdot \mathbf{K}) \boldsymbol{\mu}, \mathbf{k g} /(\mathbf{m} \cdot \mathbf{s})$ & $\boldsymbol{k}, \mathbf{W} /(\mathbf{m} \cdot \mathbf{K})$ & $\boldsymbol{P r}$ \\
\hline 0.90666 & 1012.565 & $2.2382 \cdot 10^{-5}$ & 0.03271 & 0.69287 \\
\hline
\end{tabular}

For the analyses in which air flows with the velocity, $V_{a}$ of $0.5-1 \mathrm{~m} / \mathrm{s}$ over the longitudinal fins having $10 \mathrm{~mm}$ thickness, $50 \mathrm{~mm}$ length and $300 \mathrm{~mm}$ width, the Reynolds number computed through Eq. (29) points out that the flow is laminar.

$$
\operatorname{Re}_{W}=\frac{V_{a} W}{v}
$$

That's why, laminar flow model is chosen in ANSYS Fluent. With the same parameters, CFD analyses are performed at different air velocities, which affect the convective heat transfer coefficient and thus heat transfer rate. The temperature contour graphs for air velocity of $1 \mathrm{~m} / \mathrm{s}$ are illustrated in Figure 3. a)

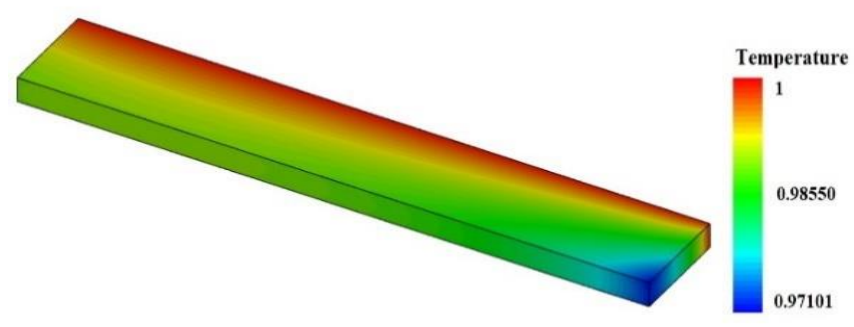

b)

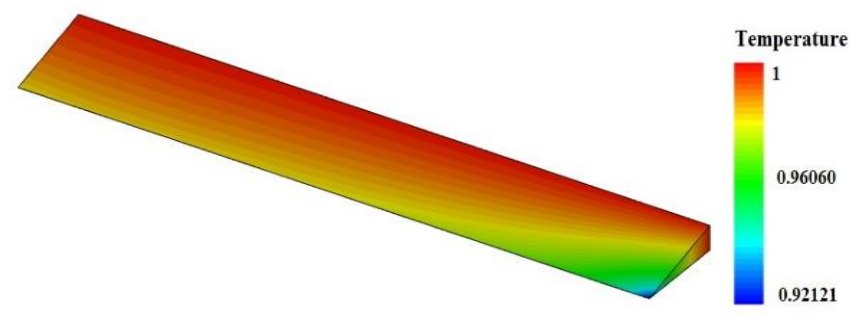

c)

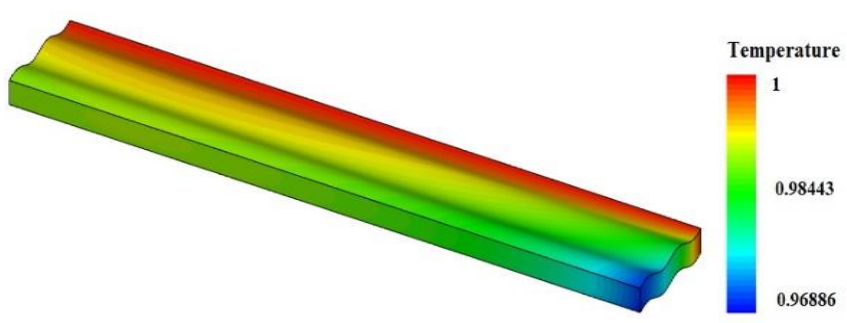

d)

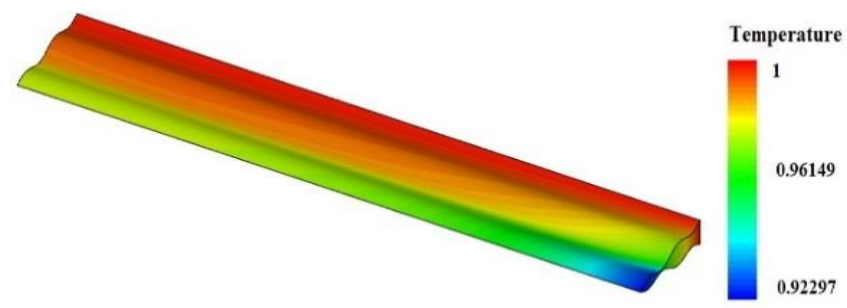

Figure 3. Temperature contours of a) rectangular b) triangular, c) wavy rectangular, d) wavy triangular fins

Heat transfer convection coefficient values are evaluated using Newton's Law of cooling.

$$
q_{f}=h A_{s}\left(T_{s}-T_{a}\right)
$$

where, $T_{s}$ is the average surface temperature. The convection coefficient values are substituted into the equation governing heat transfer rate from related fin to compare analytical and CFD results. To ensure the accuracy of the CFD results, independence of mesh must be provided. To accomplish this, the intensity of mesh in the vicinity of the fin surfaces is improved. The change of heat transfer rate and heat transfer convection coefficient with increasing number of nodes for rectangular fins is given in Table 2.

As seen in Table 2, there are minor differences between last two cases. Therefore, for the sake of saving time, the model having 765997 nodes is used. 
Table 2. The change of values with increasing number of nodes

\begin{tabular}{ccc}
\hline the number of nodes & $\mathbf{q}_{\mathbf{f}, \mathbf{W}} \mathbf{W}$ & $\boldsymbol{h}, \mathbf{W} /\left(\mathbf{m}^{2} \mathbf{K}\right)$ \\
\hline 96,406 & 34.95808 & 5.91818 \\
\hline 292,156 & 35.25281 & 5.96938 \\
\hline 765,997 & 35.63639 & 6.03463 \\
\hline $1,072,328$ & 35.69898 & 6.04527 \\
\hline
\end{tabular}

In a consequence of researches to find a similar study with the present one to validate CFD results, an appropriate study wasn't come across. However, flow over rectangular fins can be thought as flow over isothermal plates since fin material is very conductive and the magnitude of air velocities are small. Thus, Nusselt numbers obtained through CFD analysis of rectangular fins are validated with well-known Nusselt number correlations given in Eq. (31) [16] and Eq. (32) [19].

$$
\begin{aligned}
& N u=0.664 \operatorname{Re}_{W}{ }^{1 / 2} \operatorname{Pr}^{1 / 3} \\
& N u=\frac{0.6774 \operatorname{Re}_{W}{ }^{1 / 2} \operatorname{Pr}^{1 / 3}}{\left[1+(0.0468 / \operatorname{Pr})^{2 / 3}\right]^{1 / 4}}
\end{aligned}
$$

Also, it must be emphasized that the effect of radiation is not considered in the present study. The reason is that in ref [20], it was stated that the errors because of consideration of radiation only between fins and surrounding i.e. not considering fin-fin and base-fin effects, are larger than the errors because of neglection of radiation.

\section{RESULTS AND DISCUSSION}

Using Eq. (25) and Eq. (26), the numerical technique is firstly applied to triangular fins having thickness of $1 \mathrm{~cm}$, length of $5 \mathrm{~cm}$, and width of $1 \mathrm{~m}$ to obtain temperature distribution and heat transfer rate at different step sizes which are denoted by $\Delta \xi$. The results are compared with analytical ones as demonstrated in Table 3.

Table 3. The comparison of numerical and analytical results for triangular fins at $h=25 \mathrm{~W} /\left(\mathrm{m}^{2} \mathrm{~K}\right)$

\begin{tabular}{|c|c|c|c|c|}
\hline & $\Delta \xi=0.001$ & $\Delta \xi=0.0001$ & $\Delta \xi=0.00001$ & Analytical \\
\hline$\xi$ & $\Theta$, non-dimensional temperature distribution \\
\hline 0 & 0.934071897 & 0.934010640 & 0.934004511 & 0.934003856 \\
\hline 0.1 & 0.940504152 & 0.940501530 & 0.940501266 & 0.940501259 \\
\hline 0.2 & 0.947023817 & 0.947021478 & 0.947021244 & 0.947021236 \\
\hline 0.3 & 0.953566106 & 0.953564053 & 0.953563847 & 0.953563840 \\
\hline 0.4 & 0.960131070 & 0.960129305 & 0.960129128 & 0.960129121 \\
\hline 0.5 & 0.966718763 & 0.966717288 & 0.966717140 & 0.966717134 \\
\hline 0.6 & 0.973329237 & 0.973328053 & 0.973327935 & 0.973327930 \\
\hline 0.7 & 0.979962544 & 0.979961655 & 0.979961565 & 0.979961561 \\
\hline 0.8 & 0.986618739 & 0.986618144 & 0.986618084 & 0.986618082 \\
\hline 0.9 & 0.993297873 & 0.993297575 & 0.993297545 & 0.993297544 \\
\hline 1 & 0.999999999 & 0.999999999 & 0.999999999 & 1 \\
\hline \multirow{5}{|c|}{$q_{f}(\mathrm{~W})$, heat transfer rate } \\
\cline { 2 - 5 } & 422.9665776 & 422.9789004 & 422.9801352 & 422.9802813 \\
\hline
\end{tabular}

According to the results given in Table 3, more accurate results are obtained as the step size is decreased. Since the differential equation for wavy triangular fins involves much more complicated terms (trigonometric and radical expressions) the solution is performed at step sizes of 0.00001 and 0.000001 . To avoid lengthy computation time, the results are obtained at $\Delta \xi=0.00001$ since nearly same data are encountered at both step sizes. To illustrate, for $h=25 \mathrm{~W} /\left(\mathrm{m}^{2} \mathrm{~K}\right)$, tip temperature is obtained as 0.911974626 at $\Delta \xi=0.00001$ while it is 0.911974217 at $\Delta \xi=0.000001$.

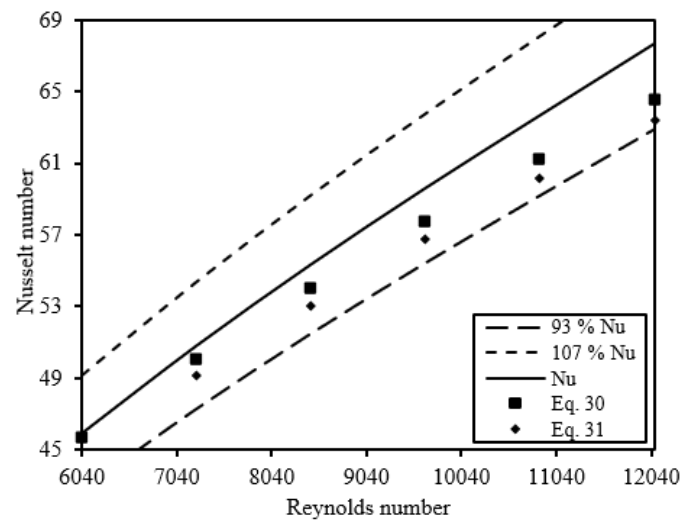

Figure 4. Nusselt number values for rectangular fins

In order to validate CFD results for rectangular fins, Nusselt number correlations given in the previous section are used. According to Figure 4, the CFD results agree well with the correlation values such that maximum difference is approximately $6.71 \%$. Also, there is a better agreement between the CFD results and the values obtained using Eq. (30) such that the maximum difference is approximately $4.77 \%$. The minimum error is obtained using Eq. (30) for $R e=6040$ with $0.59 \%$. From Figure 4, it can be easily comprehended that increasing velocity causes the difference between CFD results and correlation values to increase as well. The reason is that temperature variation within the fin becomes more noticeable with increasing velocity. Since same methodology is followed for the other examined fins in terms of mesh type and boundary conditions, the well agreement between CFD results of rectangular fins and Nusselt correlations gives an idea about validity of CFD results pertaining to other investigated fins.

As seen from Figure 5a, the difference between analytical and CFD results is about $3 \%$ at most. The situation can be explained that especially at lower air velocities, highly conductive materials used for fins leads to small temperature change throughout the thickness and flow direction of the fin, which causes high similarity between the numerical and analytical results. However, as the air velocity increases, the difference between them becomes more significant. Heat transfer from wavy rectangular fin is more than other fins while heat transfer from triangular fin is moderately less than others. The effect of sinusoidal profile on heat transfer enhancement for wavy triangular fins in comparison to wavy rectangular fins seems more observable. To illustrate, for $V_{a}=0.5 \mathrm{~m} / \mathrm{s}$, increase in heat transfer through using sinusoidal profile is $3.31 \%$ and $1.38 \%$ for triangular and rectangular fins, respectively and for $V_{a}=1 \mathrm{~m} / \mathrm{s}$, these values decrease to $3.26 \%$ and $1.332 \%$. As illustrated in Figure $5 \mathrm{~b}$, convective coefficient values are almost the same for triangular and wavy triangular fins while the values of wavy rectangular fins are slightly lower than those of rectangular fins. This slight difference causes heat dissipation values of rectangular and wavy rectangular fins to get close. This situation decreases thermal performance of wavy rectangular fins. The minor difference between convective heat transfer coefficients is due 
to the slightly different boundary layer development over different fin geometries.

a)

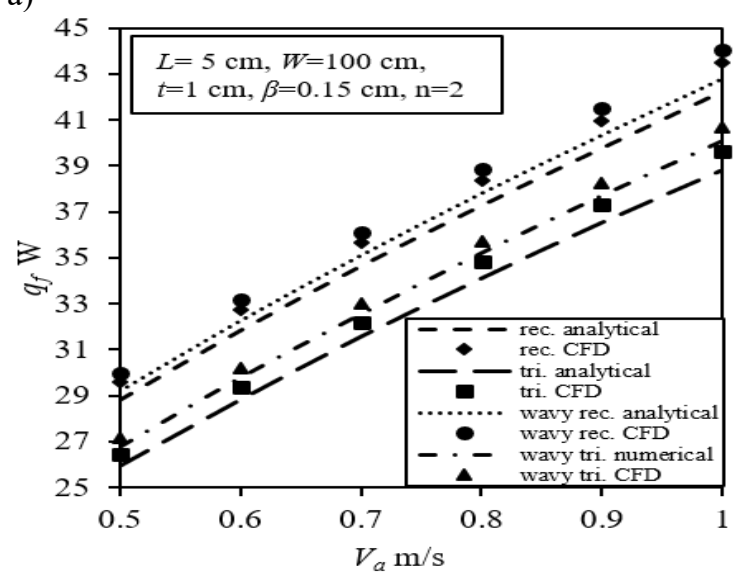

b)

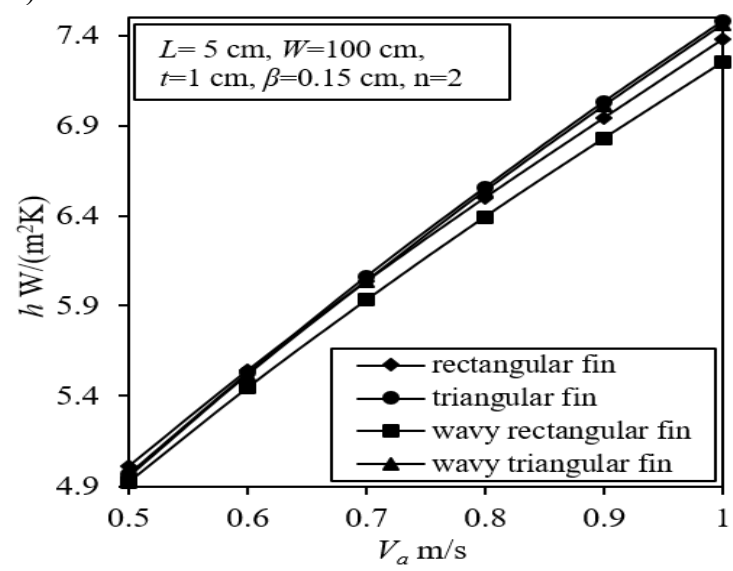

Figure 5. a) Heat transfer rates and b) convective heat transfer coefficient values

As seen in Figure 6a, the temperature distribution of the wavy triangular fins with one-wave profile does not show wavy characteristic because these fins have a profile like convex parabolic geometry, which means that they have no crests and troughs as two and three-wave triangular fins have. Therefore, there is no instant decrease in the temperature for these fins. On the other hand, temperature distribution of the wavy triangular fins with greater wave numbers demonstrates wave-like behavior. Therefore, temperature distribution behavior of wavy triangular fins is directly affected by the number of waves. Furthermore, the graph indicates that as wave number is increased, tip temperature approaches to lower values. However, in the middle regions of the wavy triangular fins having two or greater wave numbers, temperature is higher in comparison to the wavy triangular fins with onewave profile. From the results, it can be deduced that wave form contributes to temperature decrease at fin tip and regions close to the tip for wavy triangular fins. The reason is that in wavy triangular fins having two or greater wave number the cross-section gets thinner close to fin tip because of sinusoidal wave form and at this region, temperature decreases significantly. Temperature variations of the investigated fins are presented in Figure $6 \mathrm{~b}$. Temperatures at triangular and wavy triangular fins are close between 0.3 and 1 . However, there is a noticeable difference at fin tip temperature of two fins. A different relation emerges between rectangular and wavy rectangular fins such that temperature characteristics of both fins are in the same manner. Tip temperature of the wavy rectangular fin is slightly lower than that of rectangular fin. Temperature distribution of wavy rectangular fins does not show wavy characteristic because they have uniform crosssection unlike wavy triangular fins. For this reason, wavy and plate type rectangular fins are very similar in terms of temperature distribution.

a)

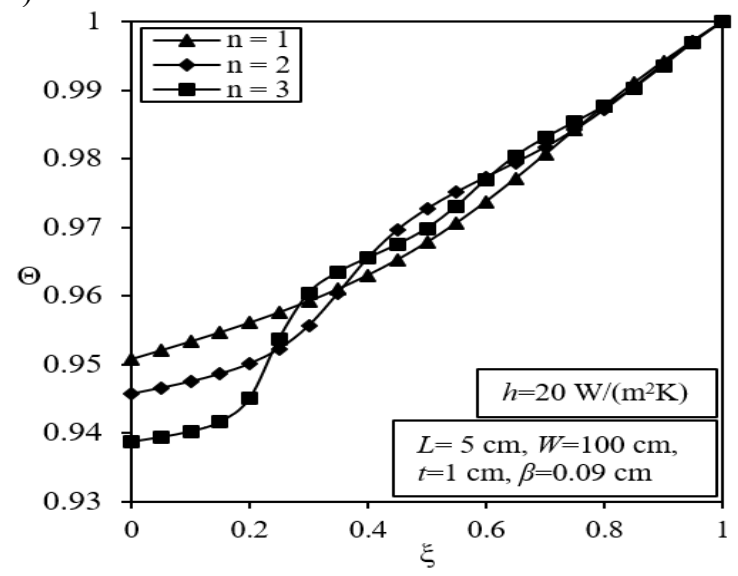

b)

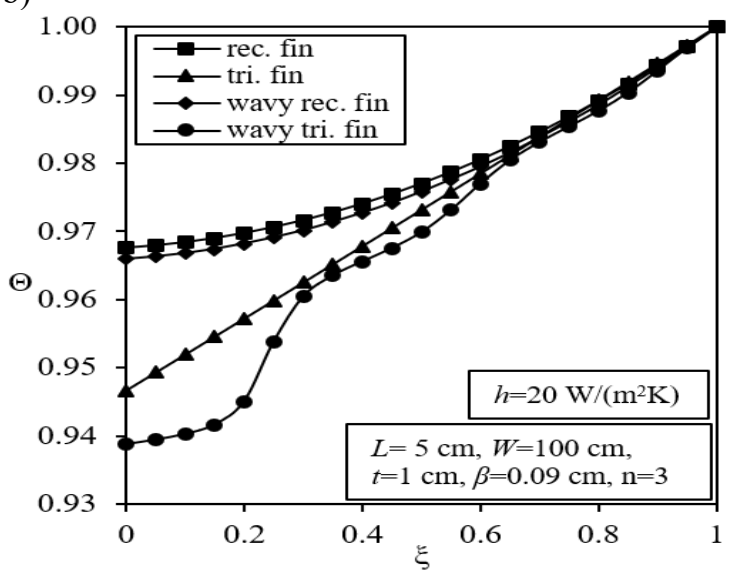

Figure 6. a) Temperature distribution of wavy triangular fins and b) comparison of temperature distributions

The performance ratio is presented in Figure $7 \mathrm{a}$ and $7 \mathrm{~b}$ for wavy rectangular and wavy triangular fins, respectively. Maximum heat transfer enhancement is provided for wavy triangular and rectangular fins by $3 \%$ and $2.5 \%$, respectively. The parameter for wavy triangular fins is larger in comparison with wavy rectangular fins for $m \cdot L=0.16-0.37$. However, the performance ratio for wavy triangular fins decreases more rapidly as the values of $m \cdot L$ increases while the change of performance ratio for wavy rectangular fins with respect to $m \cdot L$ shows flatter trend. As a result, the performance ratio of wavy triangular fins fell behind that of wavy rectangular fins beyond $m \cdot L=0.37$. Hence, it can be concluded that wavy triangular fins are more effective in terms of thermal performance for lower convective coefficients. As convective heat transfer coefficient is increased, heat transfer increases as well. However, temperature drops more suddenly, which decreases fin efficiency. Because of the significant temperature drop in wavy triangular fins, performance ratio decreases more rapidly unlike wavy rectangular fins. Another important result is that fin efficiency of rectangular fins is superior to that of wavy rectangular fins while efficiency of triangular fins is greater than that of wavy triangular fins. Also, 
it can be seen from Figure 7a that heat dissipation of wavy triangular fins with one wave goes down below that of triangular fins beyond $m \cdot L=0.47$.

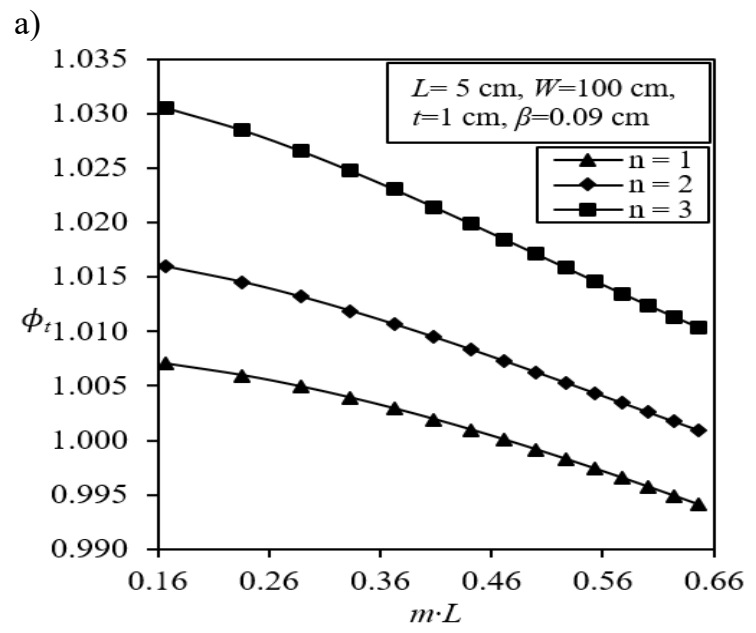

b)

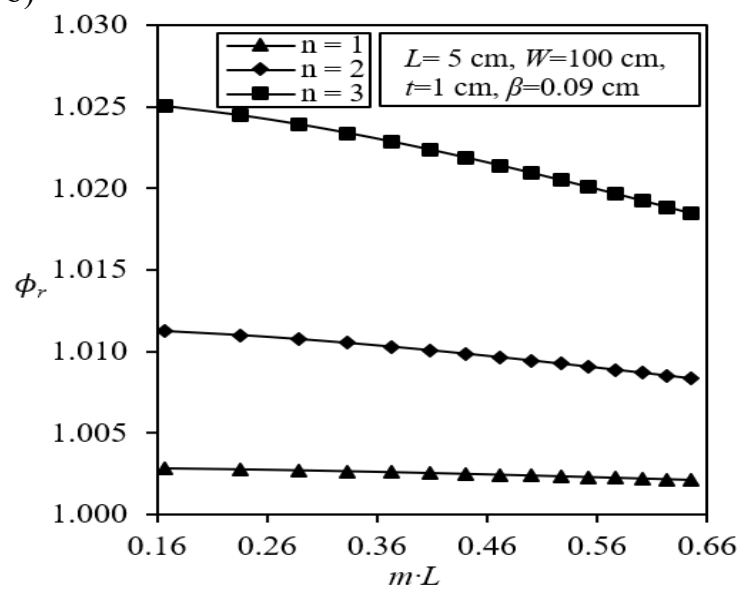

Figure 7. Performance ratio for a) wavy triangular fins and b) wavy rectangular fins

Temperature variations of triangular and wavy triangular fins are compared for the thicknesses of $1.5 \mathrm{~cm}$ and $2 \mathrm{~cm}$ in Figure $8 \mathrm{a}$ and $8 \mathrm{~b}$, respectively. Both graphs show that tip temperature of wavy triangular fins is considerably lower than that of triangular fins. The only exception occurs for wavy triangular fins with one-wave profile such that its tip temperature is higher in comparison with the tip temperature of triangular fin. In Figure 8c and 8d, the performance ratio of wavy triangular fins is presented. The results indicate that heat dissipation from wavy triangular fins significantly increases with the increasing amplitude length. For instance, the performance ratio of wavy triangular fins having one-wave profile, thickness of $2 \mathrm{~cm}$, and amplitude length of $0.21 \mathrm{~cm}$ shows approximately same result with the wavy triangular fin with three-wave profile, thickness of $1 \mathrm{~cm}$, and amplitude length of $0.09 \mathrm{~cm}$ at $m \cdot L=0.16$ as illustrated previously in Figure 7a. At the thickness of $1.5 \mathrm{~cm}$ at $m \cdot L=0.16$, about $8 \%$ increase in heat dissipation is provided while at the thickness of $2 \mathrm{~cm}$, the increase in heat transfer becomes $15.3 \%$. As seen from Figure 8c-d, the performance ratio decreases with $m \cdot L$ values more slightly in comparison to the situation given in Figure $7 \mathrm{~b}$. The reason is that the increase in heat transfer due to increased surface area tolerates the significant temperature drop. a)

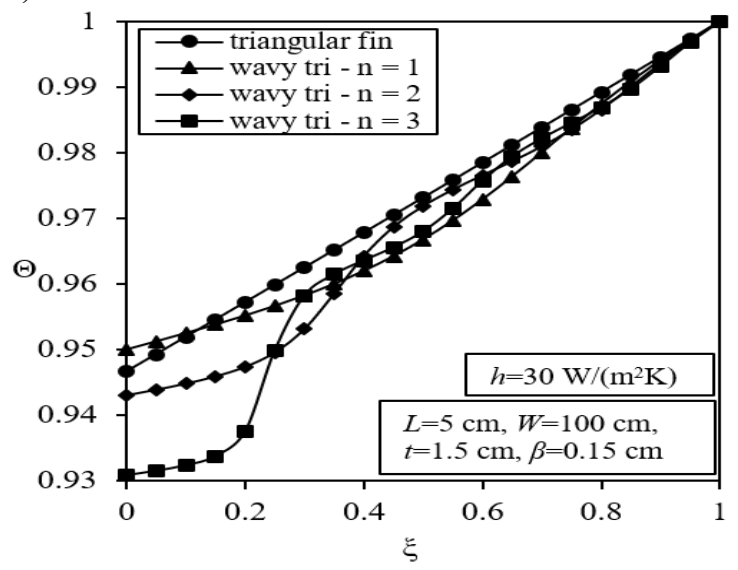

b)

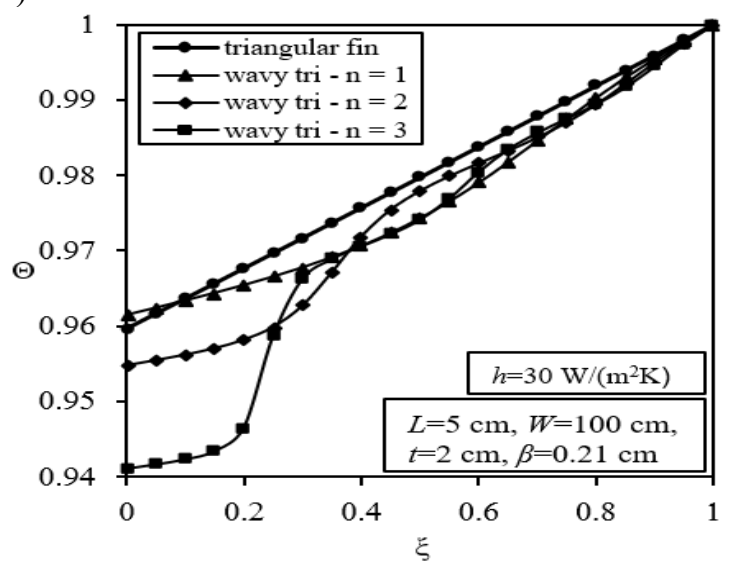

c)

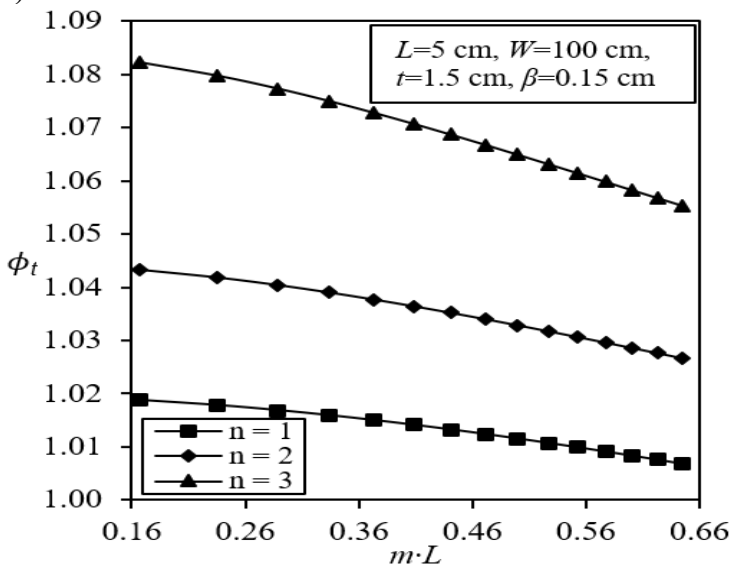

d)

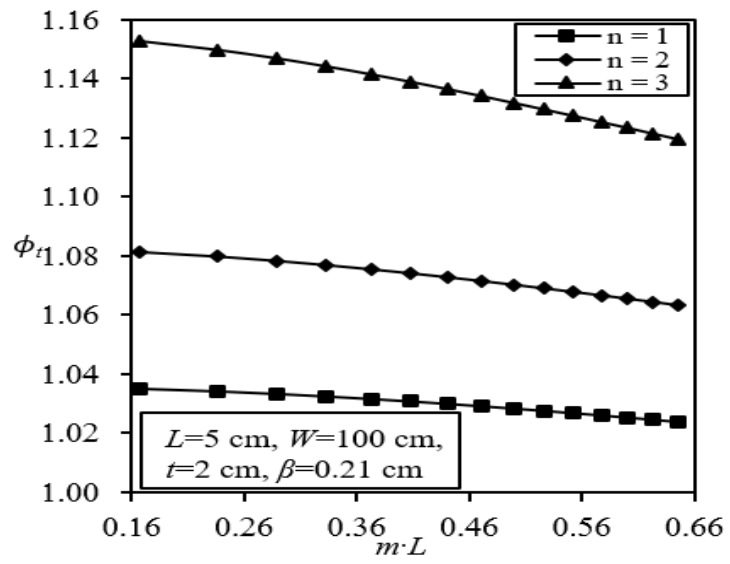

Figure 8. Temperature distribution of triangular and wavy triangular fins a) $t=1.5 \mathrm{~cm} \mathrm{~b}) t=2 \mathrm{~cm}$, performance ratio of wavy triangular fins for c) $t=1.5 \mathrm{~cm} \mathrm{~d}) t=2 \mathrm{~cm}$ 


\section{CONCLUSION}

In the present study, different longitudinal fins are investigated analytically and numerically. One part of the numerical analysis involves the solution of the governing equation of wavy triangular longitudinal fins. The Shooting and Cash-Karp Runge Kutta Methods are invoked for the solution. The latter part of the numerical analysis is conducted using CFD at different air velocities. In the light of the results, the outcomes are summarized as follows:

- Both CFD results and analytical results show that use of sinusoidal profile in triangular fins have better effects on thermal performance in comparison to wavy rectangular fins. Also, CFD results shows that between the air velocity of $0.5-1 \mathrm{~m} / \mathrm{s}$, lowest heat transfer convection coefficient values belong to wavy rectangular fins.

- Among the investigated fins, the lowest temperature values at the fin tip are obtained for wavy triangular fins. At the same amplitude length, with increasing wave number, temperature decrease in wavy triangular fins become more significant. Moreover, temperature decrease in wavy rectangular fins having three waves and amplitude length of $0.09 \mathrm{~cm}$ is almost same with rectangular fins.

- When amplitude length of wavy triangular fins is increased, considerable improvement in heat transfer rate is obtained such that heat dissipation increases by $15.3 \%$ at the amplitude length of $0.21 \mathrm{~cm}$ and three waves.

In conclusion, wavy triangular fins are modelled mathematically, and the resulting differential equation is solved numerically. Thus, temperature variation and heat transfer performance of this new fin type are investigated, and the results are compared with other three fin configurations. Furthermore, CFD analyses of the fins are performed to accomplish a realistic approach.

\section{REFERENCES}

[1] Bejan, A., Kraus, A.D. (2003). Heat Transfer Handbook. New Jersey, John Wiley \& Sons, 201-215.

[2] Kraus, A.D., Aziz, A., Welty, J. (2002). Extended Surface Heat Transfer. New York, John Wiley \& Sons, $1-58$.

[3] Khaled, A.R.A., Gari, AA. (2014). Heat transfer enhancement via combined wall and triangular rooted-fin system. Journal of Electronics Cooling and Thermal Control, 4(1): 12-21.

[4] Khaled, A.R.A. (2010). Thermal characterizations of exponential fin systems. Mathematical Problems in Engineering, 2010: 1-19. http://dx.doi.org/10.1155/2010/765729

[5] Turkyilmazoglu, M. (2012). Exact solutions to heat transfer in straight fins of varying exponential shape having temperature dependent properties. International Journal of Thermal Sciences, 55: 69-75. http://dx.doi.org/10.1016/j.ijthermalsci.2011.12.019

[6] Khaled, A.R.A. (2015). Thermal performance of six different types of wavy-fins. International Journal of Numerical Methods for Heat \& Fluid Flow, 25(4): 892911. http://dx.doi.org/10.1108/HFF-06-2014-0174

[7] Lotfi, B., Abdellah, B. (2014). Numerical method for optimum performance of fin profiles. International Journal of Current Engineering and Technology, 4(6): 3990-3998.
[8] Altun, A.H., Ziylan, O. (2019). Experimental investigation of the effects of horizontally oriented vertical sinusoidal wavy fins on heat transfer performance in case of natural convection. International Journal of Heat and Mass Transfer, 139: 425-431. http://dx.doi.org/10.1016/j.ijheatmasstransfer.2019.05.0 09

[9] Nilpueng, K., Ahn, H.S., Jerng, D.W., Wongwises, S. (2019). Heat transfer and flow characteristics of sinusoidal wavy plate fin heat sink with and without crosscut flow control. International Journal of Heat and Mass Transfer, 137: 565-572. http://dx.doi.org/10.1016/j.ijheatmasstransfer.2019.03.1 14

[10] Sevilgen, G. (2015). A numerical analysis of a convective straight fin with temperature-dependent thermal conductivity. Thermal Science, 21(2): 939-952. http://dx.doi.org/10.2298/TSCI141201055S

[11] Silva, M.J., Zdanski, P.S.B., Vaz, J.M. (2017). Forced convection on grey cast iron plate-fins: Prediction of the heat transfer coefficient. International Communications in Heat and Mass Transfer, 81: 1-7. http://dx.doi.org/10.1016/j.icheatmasstransfer.2016.11.0 07

[12] Torabi, M., Zhang, Q. (2013). Analytical solution for evaluating the thermal performance and efficiency of convective-radiative straight fins with various profiles and considering all non-linearities. Energy Conversion and Management, 66: 199-210.

[13] Torabi, M., Aziz, A., Zhang, K. (2013). A comparative study of longitudinal fins of rectangular, trapezoidal and concave parabolic profiles with multiple nonlinearities. Energy, 51: 243-256.

[14] Cortell, R. (2008). A numerical analysis to the non-linear fin problem. Journal of Zhejiang University-SCIENCE A, 9(5): 648-653. http://dx.doi.org/10.1631/jzus.A0720024

[15] Zaimi, W.M.K.A.D., Bidin, B., Bakar, N.A.A., Hamid, R.A. (2012). Applications of Runge-Kutta-Fehlberg method and Shooting technique for solving classical Blasius equation. World Applied Sciences Journal, 17: 10-15.

[16] Incropera, F.P., DeWitt, D.P., Bergman, T.L., Lavine, AS. (2013). Principles of Heat and Mass Transfer, New Jersey, John Wiley \& Sons, 154-178.

[17] Cash, J.R., Karp, A.H. (1990). A variable order RungeKutta method for initial value problems with rapidly varying right-hand sides. ACM Transactions on Mathematical Software (TOMS), 16(3): 201-222. http://dx.doi.org/10.1145/79505.79507

[18] Versteeg, H.K., Malalasekera, W. (2007). An introduction to computational fluid dynamics: The finite volume method. London, Pearson Education, 9-39.

[19] Churchill, S.W., Ozoe, H. (1973). Correlations for laminar forced convection with uniform heating in flow over a plate and in developing and fully developed flow in a tube. Journal of Heat Transfer, 95(1): 78-84. http://dx.doi.org/10.1115/1.3450009

[20] Khor, Y.K., Hung, Y.M., Lim, B.K. (2010). On the role of radiation view factor in thermal performance of straight-fin heat sinks. International Communications in Heat and Mass Transfer, 37(8): 1087-109. http://dx.doi.org/10.1016/j.icheatmasstransfer.2010.06.0 12 


\section{NOMENCLATURE}

$\mathrm{A}_{\mathrm{c}} \quad$ cross-sectional area, $\mathrm{m}^{2}$

$\mathrm{A}_{\mathrm{s}} \quad$ surface area, $\mathrm{m}^{2}$

$\mathrm{c}_{\mathrm{p}} \quad$ specific heat, $\mathrm{J}_{\mathrm{kg}}{ }^{-1} \cdot \mathrm{K}^{-1}$

$\mathrm{F}_{\mathrm{r}} \quad$ profile function for wavy rectangular longitudinal

$F_{r} \quad$ fin, $m$

$\mathrm{F}_{\mathrm{t}} \quad$ profile function for wavy triangular longitudinal fin,

$\mathrm{h} \quad \mathrm{m}$ convective heat transfer coefficient, W. $\mathrm{m}^{2} . \mathrm{K}$

$\mathrm{k}$ thermal conductivity, $\mathrm{W} \cdot \mathrm{m}^{-1} . \mathrm{K}$

$\mathrm{L} \quad$ fin length, $\mathrm{m}$

$\mathrm{m} \quad$ fin performance factor, $\mathrm{m}^{-1}$

$\mathrm{n}$ the number of waves

$\mathrm{Nu}$ the average Nusselt number

$\mathrm{q}_{\mathrm{f}}$ heat dissipation from fin, $\mathrm{W}$

$\mathrm{Re}_{\mathrm{w}} \quad$ Reynolds number

$\mathrm{S}_{\mathrm{r}}$ the arc length of sinusoidal profile for wavy rectangular fin, $\mathrm{m}$

$\mathrm{S}_{\mathrm{t}}$ the arc length of sinusoidal profile for wavy triangular fin, $\mathrm{m}$

$\mathrm{t}$ fin thickness, $\mathrm{m}$

$\mathrm{T}$ temperature at any point of fin, ${ }^{\circ} \mathrm{C}$

$\mathrm{T}_{\mathrm{a}} \quad$ ambient temperature, ${ }^{\circ} \mathrm{C}$
$\mathrm{T}_{\mathrm{b}} \quad$ fin base temperature, ${ }^{\circ} \mathrm{C}$

$\mathrm{T}_{\mathrm{f}} \quad$ film temperature, ${ }^{\circ} \mathrm{C}$

$\mathrm{T}_{\mathrm{s}} \quad$ surface temperature, ${ }^{\circ} \mathrm{C}$

$\mathrm{V}_{\mathrm{a}} \quad$ free stream velocity, $\mathrm{m} . \mathrm{s}^{-1}$

W fin width, $m$

w temperature gradient in the numerical solution, K.m ${ }^{-1}$

\section{Greek symbols}

$\beta \quad$ length of amplitude, $m$

$\delta$ dimensionless coordinate following the sinusoidal profile

$\Delta \xi \quad$ step size

$\Theta \quad$ dimensionless temperature

$\mu \quad$ dynamic viscosity, $\mathrm{kg} \cdot \mathrm{m}^{-1} \cdot \mathrm{s}^{-1}$

$v \quad$ kinematic viscosity, $\mathrm{m}^{2} \cdot \mathrm{s}^{-1}$

$\xi \quad$ dimensionless axial coordinate

$\rho \quad$ density, $\mathrm{kg} \cdot \mathrm{m}^{-3}$

$\tau \quad$ time, $\mathrm{s}$

$\Phi_{r} \quad$ performance ratio of wavy rectangular longitudinal

$\Phi_{\mathrm{r}} \quad$ fin

$\Phi_{\mathrm{t}} \quad$ performance ratio of wavy triangular longitudinal fin 